\title{
Hippocampal inactivation enhances taste learning
}

\author{
Martha E. Stone, Brandon S. Grimes, and Donald B. Katz ${ }^{1}$ \\ Psychology Department and Volen National Center for Complex Systems, Brandeis University, \\ Waltham, Massachusetts 02454-9110, USA
}

\begin{abstract}
Learning tasks are typically thought to be either hippocampal-dependent (impaired by hippocampal lesions) or hippocampal-independent (indifferent to hippocampal lesions). Here, we show that conditioned taste aversion (CTA) learning fits into neither of these categories. Rats were trained to avoid two taste stimuli, one novel and one familiar. Muscimol infused through surgically implanted intracranial cannulae temporarily inactivated the dorsal hippocampus during familiarization, subsequent CTA training, or both. As shown previously, hippocampal inactivation during familiarization enhanced the effect of that familiarization on learning (i.e., hippocampal inactivation enhanced latent inhibition of (TA); more novel and surprising, however, was the finding that hippocampal inactivation during training sessions strongly enhanced CTA learning itself. These phenomena were not caused by specific aspects of our infusion technique-muscimol infusions into the hippocampus during familiarization sessions did not cause CTAs, muscimol infusions into gustatory cortex caused the expected attenuation of CTA, and hippocampal inactivation caused the expected attenuation of spatial learning. Thus, we suggest that hippocampal memory processes interfere with the specific learning mechanisms underlying CTA, and more generally that multiple memory systems do not operate independently.
\end{abstract}

With few exceptions, learning tasks are thought to be either "hippocampal-dependent" (i.e., impaired by hippocampal lesion) or "hippocampal-independent" (i.e., unaffected by hippocampal lesion) (for review, see Eichenbaum 2004). Conditioned taste aversion (CTA), a form of learning in which a normally appetizing taste becomes aversive following its association with gastric distress, requires a neural circuit that includes the pons, taste cortex, and amygdala (Gallo et al. 1992; Spector 1995; Lamprecht et al. 1997; Yasoshima and Yamamoto 1997; Schafe et al. 1998) and is generally thought to be hippocampal-independent (Yamamoto et al. 1995; Bures et al. 1998).

Interpretation of the data suggesting that CTA is a hippocampal-independent form of learning is complicated, however, by the fact that all of the above-mentioned studies used permanent lesions. Such lesions have complex effects, downregulating some systems and upregulating others; during the 1-2 wk of recovery that must pass before rats with permanent lesions are healthy enough to participate in experiments, these changes evolve in a way that may extend, mask, or compensate for acute loss of hippocampal activity (e.g., Hannigan Jr. et al. 1984; Simon et al. 1988; Lipska et al. 1992). Because particular parts of the systems that interact with the hippocampus are deeply involved in sensory aspects of CTA (Reilly and Bornovalova 2005), it is possible that temporary hippocampal inactivations might have an effect, even a facilitory one, on CTA, despite the lack of such effects observed on the majority of tasks.

Another reason to hypothesize that taste learning in particular might actually be facilitated by loss of the hippocampus has to do with latent inhibition-the process whereby familiarization with a taste reduces later learning of a CTA for that taste. Latent inhibition of CTA itself appears to be enhanced by hippocampal lesions (Reilly et al. 1993; Gallo and Candido 1995; Purves et al. 1995), despite the fact that in most paradigms latent inhibition is impaired by damage to the hippocampus or related structures (Kaye and Pearce 1987; Schmajuk et al. 1994; Yee et al.

\footnotetext{
'Corresponding author.

E-mail dbkatz@brandeis.edu; fax (781) 736-2398.

Article and publication are at http://www.learnmem.org/cgi/doi/10.1101/ Im.32305.
}

1995; Coutureau et al. 1999; Grecksch et al. 1999; Shohamy et al. 2000). Such data suggest that the role of hippocampus in learning may depend upon the specific paradigm and system being studied. Related work suggests that hippocampal spatial learning processes interfere with striatal-dependent "habit learning" processes, such that habit learning is enhanced following hippocampal lesions (Schroeder et al. 2002; Poldrack and Packard 2003). It is possible that a similar enhancement of amygdala-dependent CTA learning may follow removal of the hippocampus from the circuit, although such a phenomenon has never before been reported.

Here we replicate the finding that removal of the hippocampus enhances latent inhibition of CTA, and further show that temporary, muscimol-induced inactivation of the dorsal hippocampus does in fact enhance CTA: Rats with inactivated hippocampi learn stronger aversions than normal rats. Identical inactivations caused the expected impairment of spatial learning, and further control experiments/analyses demonstrated that this result reflects neither specific aspects of our training regimen nor of muscimol infusion. Based on these data, we conclude that hippocampal learning processes interfere with those more centrally involved in CTA, and further suggest that latent inhibition circuits are to some degree separate from basic CTA circuits. At a more general level, our data serve as strong evidence that hippocampal and amygdalar learning circuits are not independent.

\section{Results}

Figure 1 presents a highly representative coronal slice through the dorsal hippocampus (dHIPP) in a randomly selected rat that received muscimol (MUS) infusions during CTA conditioning sessions. The guide and infusion cannulae descend through cortex and end in the CA1 cell fields of dHIPP. Figure 1B shows a schematic, taken from Paxinos and Watson (1997), in which arrows point to the boxed regions found to contain all guide cannula tips. While it is difficult to estimate the precise spread of MUS or any non-lesioning agent, our slowly delivered infusion into dHIPP, just below and around the cannula tips, is almost certainly responsible for the effects described below, and also makes it clear that the damage caused by the 23-gauge guide 


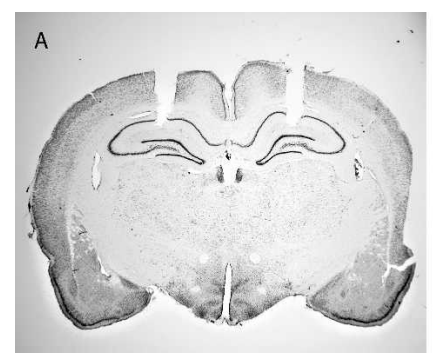

B

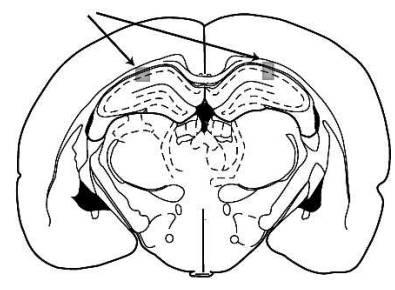

Figure 1. Infusion cannulae were located in the CA1 field of the dorsal hippocampus. (A) Photomicrograph of a coronal slice through the dorsal hippocampus of a representative (i.e., randomly selected) rat. The cannula tracks are clearly visible, descending into the CA1 region of the dorsal hippocampus. (B) Schematic (reprinted with permission from Elsevier ( $)$ 1997, from Paxinos and Watson 1997) of tip locations (gray boxes, indicated by arrows).

cannulae (accentuated by slight tissue shrinkage during processing) had little impact on our results.

We set out to examine whether CTA is enhanced by temporary dHIPP inactivation. Our "within-subject" protocol also enabled us to replicate previously demonstrated lesion-induced enhancements of latent inhibition (Purves et al. 1995); each rat was trained to avoid two different tastes, one familiar (taste-fam) and one novel (taste-nov). Because the results of Purves et al. were essentially identical to those demonstrated in a between-group procedure (Reilly et al. 1993), it can be assumed that the withingroup procedure did not cause undue changes to the learning processes involved.

The tastes used were $0.1 \mathrm{M}$ sucrose and $0.1 \mathrm{M} \mathrm{NaCl}$ (counterbalanced for familiarity). For one of five groups, MUS was infused into dHIPP just before the four familiarization sessions with taste-fam, and vehicle (VEH) was infused just before the four conditioning sessions with taste-fam/taste-nov followed by intraperitoneal $\mathrm{LiCl}$ injections. The second group received the opposite treatment, the third group received MUS in all familiarization and training sessions, the fourth (control) group received $\mathrm{VEH}$ in all sessions, and the fifth group was an unoperated control that received no infusions. In all cases, the behavior of unoperated controls was not statistically different from that of any conditioning involving only VEH infusion.

The amount (in $\mathrm{mL}$ ) of fluid consumed in the sole testing sessions by rats that received MUS infusions during familiarization or training sessions (but not both, see below) is shown in Figure 2, along with control data. The success of the familiarization sessions in producing latent inhibition is clear because rats consistently drank less taste-nov than taste-fam.

Hippocampal inactivation had two main impacts on learning, both driving down consumption of taste-nov and driving up consumption of taste-fam. Rats that received MUS infusions just before training sessions developed stronger than normal CTAs; that is, they learned to drink less taste-nov than rats that received infusions of VEH (or nothing). Rats that received MUS infusions during familiarization sessions, meanwhile, drank more tastefam than VEH/control rats; MUS enhanced latent inhibition of CTA.

These effects were borne out in a two-way ANOVA for infusant (MUS, VEH/nothing) and taste (taste-fam, taste-nov), which showed a significant effect of taste $\left(F_{(1,60)}=25.54\right.$, $P<0.00001)$ and a significant interaction between taste and infusant $\left(F_{(1,60)}=5.50, P=0.02\right)$. Subsequent analysis revealed that MUS reduced drinking of taste-nov (Scheffe $F^{\prime}=4.80, P<0.001$ ) but increased drinking of taste-fam (Scheffe $F^{\prime}=2.79, P=0.03$ ). To further probe this latter effect, which we suspect was limited by a ceiling on within-session drinking, we compared both MUS- and VEH-taste-fam groups to the unconditioned, NaCl-injected controls: The MUS-taste-fam rats proved indistinguishable from the ceiling provided by controls $\left(t_{(13)}=1.1, P=0.28\right)$, while VEHtaste-fam rats drank significantly less than controls $\left(t_{(22)}=3.74\right.$, $P<0.002)$. Hippocampal inactivation during familiarization sessions enhanced latent inhibition (i.e., increased drinking in testing sessions) to the largest degree possible in our paradigm.

Differences in taste learning may sometimes be the result of differences in initial drinking; a rat that drinks less of a taste during a conditioning session (for instance, perhaps because a hippocampal infusion reduced desire for fluids) will later prove to have learned less about that taste. In anticipation of this possibility, we ensured that our rats had less to drink than they desired, such that all drank the same amount. Each and every rat drank the full $10 \mathrm{~mL}$ of fluid in $<30 \mathrm{~min}$, regardless of condition. Clearly, any small effect that hippocampal MUS infusions had on normal drinking behavior did not interfere with rats drinking the full amount available. Therefore, learning differences between the groups do not reflect differential exposure to either taste. These data also suggest that intracranial infusions of MUS do not themselves cause CTA; a single pairing of taste and LiCl-induced illness was sufficient to reduce consumption of $\mathrm{NaCl}$ or sucrose to $\sim 6 \mathrm{~mL}$ (data not shown), but rats never drank less than a full $10 \mathrm{~mL}$ in the familiarization sessions, even following a previous pairing of taste-fam and muscimol infusion.

It might also be argued that some aspect of the two-taste paradigm caused the observed effects. Perhaps the two tastes "competed" for associability with illness, such that a MUS effect on taste-fam affected learning to taste-nov (and vice versa). The data, however, do not suggest that such between-taste interaction occurred. For instance, two groups-unoperated controls, and rats that received MUS during familiarization but VEH during conditioning-had similar experiences with taste-nov but different experiences with taste-fam. Any between-taste interaction would have been apparent in a difference between the two groups in taste-nov consumption. No such difference was found $(P>0.1)$. More generally, there is no significant correlation between consumption of taste-nov and taste-fam by single rats

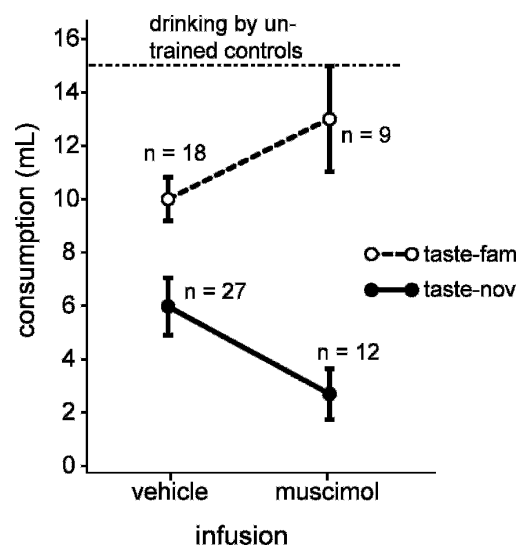

Figure 2. MUS infusion enhances both CTA and latent inhibition of CTA. (x-axis) Infusant (MUS or VEH), ( $y$-axis) $\mathrm{mL}$ of tastant consumed during testing sessions. Latent inhibition is apparent in the fact that rats always drank less taste-nov (filled circles, solid lines) than they did tastefam (open circles, dashed lines). MUS increased consumption of tastefam and decreased consumption of taste-nov (note the lack of parallelism in the lines); that is, MUS infusion during training enhanced CTA, and MUS during familiarization enhanced latent inhibition. The dot-dash line across the top is the amount drunk by a group $(n=6)$ of shamconditioned rats in testing session 1 , which did not differ from the amount of taste-fam consumed following MUS infusions. Error bars here (and in all figures) are standard errors of the mean. 
$\left(r^{2}=0.05\right.$ once the lack of normality in consumption of taste-nov was corrected; $\mathrm{r}^{2}=0.19$ before; Fig. 3 ); a strong correlation would be expected if tastes had competed for associability.

Previous studies examining the issue using permanent lesions have found that the hippocampus plays no role in CTA (Yamamoto et al. 1995; Bures et al. 1998); even the studies that previously reported enhancements of latent inhibition following permanent hippocampal lesions, including the one on which we based our protocol (Purves et al. 1995), did not report enhancements of CTA (Reilly et al. 1993). Therefore, we ran an additional group of rats in which we more closely mimicked the permanent lesions used by Purves et al. and Reilly et al.: These rats received MUS infusions before all four familiarization sessions and all four conditioning sessions. This group could be thought of as a "virtual lesion" group, in that rats received dHIPP inactivation before every training session. (To make the analogy complete, of course, rats would have to receive MUS infusions before the testing sessions, as well, which we did not do).

Figure 4 presents the amount of taste-nov drunk in testing sessions by rats that had received either MUS in all previous sessions or VEH in all previous sessions. These data demonstrate that MUS infusions significantly enhanced learning of an aversion to taste-nov (directional $t_{(24)}=1.71, P=0.05$ ). MUS infusion during all familiarization and training sessions did not increase latent inhibition for taste-fam (data not shown), but this was as expected: dHIPP inactivation during familiarization sessions should increase consumption (i.e., enhance latent inhibition), and dHIPP inactivation during training sessions should reduce consumption (i.e., enhance learning); the two effects cancel each other out.

While our data suggest that the inactivation-induced enhancement of CTA was not due to any LiCl-like effect of the MUS itself, it remains possible that some tendency for taste-MUS pairings to lead to CTA was masked by the 10 -mL cap on drinking in familiarization sessions. To provide further evidence that this was not the case, and to test the reliability of our training and infusion procedures, we prepared a subset of rats with cannulae in the primary gustatory cortex, a brain region known to be required for normal CTA learning (Gallo et al. 1992; Naor and Dudai 1996; Gutierrez et al. 2003). We trained these rats on a simplified version of the regimen (see Materials and Methods), infusing either MUS or VEH into cortex during conditioning sessions. We used $0.02 \mathrm{M}$ citric acid, a solution that the rats were willing to drink but do not normally prefer over water, as the conditioned taste, because a subset of these rats had already been exposed to both sweet and salty tastes.

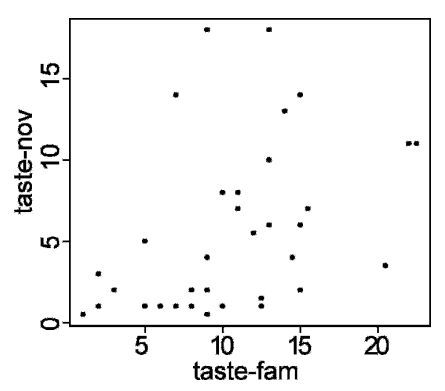

Figure 3. Lack of competition between taste-nov and taste-fam. Within-rat analysis of amount consumed during testing sessions, with taste-fam on the $x$-axis and taste-nov on the $y$-axis. The fact that most points fall in the lower right half of the space reflects latent inhibitiongreater consumption of taste-fam. The lack of an obvious relationship between the two variables (which is insignificant when the non-normality of taste-nov consumption is controlled for) makes it clear that the two probably did not compete for associability. See text for details.

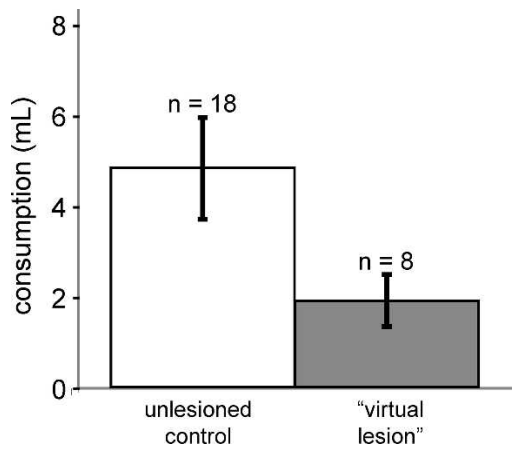

Figure 4. Enhanced CTA could be seen even when MUS was infused in all familiarization and training sessions. The $y$-axis shows $\mathrm{mL}$ of taste consumed during the testing sessions ( $x$-axis) for rats receiving MUS ("virtual lesion") or VEH ("unlesioned control") infusions across the entire training regimen-familiarization sessions with one taste, and training sessions with both tastes. MUS infusions before conditioning sessions decreased consumption of taste-nov.

Figure 5 presents fluid consumption for rats with and without cortical inactivation during the sole testing session, as well as consumption by untrained rats that received unpaired presentations of the taste and $\mathrm{LiCl}$. Far from enhancing learning, singlesite cortical inactivation during the conditioning session was enough to totally disrupt learning of the aversion to mild citric acid $\left(t_{(11)}=4.30, P<0.001\right)$, consistent with the results of many previous studies (Lasiter et al. 1985; Gallo et al. 1992; Yamamoto et al. 1995; Bielavska and Roldan 1996; Naor and Dudai 1996; Schafe and Bernstein 1998). We conclude that our infusion technique was reliable and robust: Rats learned a normal, cortically dependent aversion in our paradigm, and MUS infusions did not, in and of themselves, lead to enhanced taste learning. Only MUS infusions into dHIPP enhanced CTA.

Finally, we addressed the possibility that our infusions were not specific to dHIPP. In a control experiment, we tested the efficacy of our hippocampal infusions by examining performance of dHIPP rats on a reliably hippocampal-dependent spatial learning task, the Morris Water Maze task, in which rats must learn the relationships between distal spatial cues to find a submerged platform in a pool of milky water. Water maze learning has been shown to be impaired by dHIPP lesion (Bannerman et al. 1999), and performance deficits in this task are remarkably similar regardless of whether dHIPP is removed via excitotoxic lesions or single-site infusions of muscimol (Moser and Moser 1998). We replicated the latter study with rats prepared as for the CTA experiments, testing retention and new learning with intact and inactivated hippocampi.

Figure 6A shows the basic learning curves before surgery. Rats learned to find the submerged platform across 16 trials, after which performance asymptoted. This learning is also reflected in probe trials without a platform (Fig. 6B), which caused rats to preferentially search the quadrant of the pool in which the platform should have been. Here, we also show that the performance of rats that later received MUS infusions (dark bars) was indistinguishable from that of other rats (light bars).

Figure $6 \mathrm{C}$ reveals that dHIPP inactivation had the expected impact on retention $1 \mathrm{wk}$ following training and surgery. Control rats retained the learning, in that they continued to spend much more time in the "appropriate" quadrant than would be predicted by chance $(25 \%)$. The performance of rats that had received MUS infusions into dHIPP, meanwhile, did not differ from chance. MUS had an even stronger impact on relearning, as shown in the results from the third probe trial in Figure 6D: Control rats learned to spend their time where they expected the 


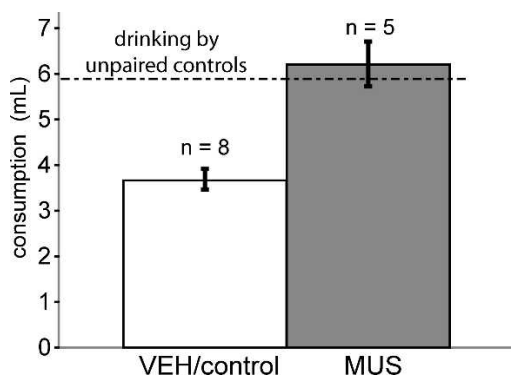

Figure 5. MUS in gustatory cortex impairs CTA. The $y$-axis shows $\mathrm{mL}$ of citric acid consumed by rats receiving MUS infusion or VEH/no infusion. As expected, MUS infusion doubled the amount of tastant consumed, eliminating CTA to the citric acid (MUS rats consumed as much as unpaired controls, consumption of which is shown by the horizontal dashed line).

platform to be, whereas rats with inactivated hippocampi did not (two-tailed $t_{(6)}=2.75, P=0.03$ ). Clearly, our hippocampal inactivations impaired spatial learning.

\section{Discussion}

It is traditional to think of hippocampal memory circuits as working in relative independence from other memory systems (see below for the sole exception). Here, however, we have shown that both CTA and latent inhibition of CTA are enhanced by reversible hippocampal inactivation. Stated simply, hippocampal inactivation improves taste learning.

Several possible confounds can be ruled out as causes of this learning enhancement: (1) all rats drank the same amount during familiarization, and thus differences in the volume of preexposure cannot explain our results; (2) inactivation of dHIPP during familiarization sessions 1-4 did not lead to reduced drinking, suggesting that intra-hippocampal MUS infusions did not, in and of themselves, cause CTA; (3) identically delivered inactivation of gustatory cortex had the expected, previously observed effect on CTA, demonstrating that our training and infusion techniques worked appropriately and proving conclusively that intracranial MUS infusions do not ubiquitously cause or enhance CTA; (4) MUS infusion into dHIPP had the expected, previously observed effect on spatial learning and performance, demonstrating that we were in fact inactivating hippocampal neurons; and (5) learning was specifically enhanced for rats that underwent a change from an altered "state" (i.e., dHIPP inactivation) during conditioning to a normal "state" during testing, which implies that "state-dependent learning", according to which changes in state should impair learning (Santini et al. 2001; Bast et al. 2003), cannot explain our findings.

The learning enhancement that accompanied hippocampal inactivation was unexpected-it has repeatedly been reported that permanent hippocampal lesions have no impact on CTA (Reilly et al. 1993; Purves et al. 1995; Yamamoto et al. 1995; Bures et al. 1998). Two factors probably contribute to the difference between our results and those reported previously. First, aversions expressed in our experiment were smaller than those typically induced. Often, taste aversion conditioning causes rats to nearly cease consumption of the taste, a ceiling effect for learning that makes further enhancements difficult to detect. Our rats, meanwhile, were supplied with only one lick spout (which strongly encouraged at least some consumption in our thirsty rats, even of a taste which to them was aversive) and were trained in a relatively complex, two-tastant training protocol; this combination of factors likely led them to express a relatively small reduction of consumption to even the novel taste, a fact that made our paradigm more sensitive to enhancements of learning.
Second, our temporary inactivations probably had fewer secondary effects on learning-related systems than do the more commonly used permanent lesion techniques. During the 1-2 weeks of recovery that must necessarily pass before rats with permanent lesions recover enough to participate in experiments, secondary extra-hippocampal changes emerge, changes that may mask or compensate for the acute effects of hippocampal removal (see below). It is entirely possible that a learning enhancement induced by hippocampal inactivation, such as is apparent in our data set, could vanish across such a time period.

While it is possible to argue that our results could be specific to a two-stimulus paradigm, several facts make such an explanation unlikely. First, the use of multiple-stimuli paradigms has a long history in behavioral neuroscience (e.g., Hall and Minor 1984; Bellingham et al. 1985; Kehoe et al. 1993) and is generally thought to involve the same processes as single-stimulus training. Second, the results of the within-subject, permanent lesion experiment upon which we based our paradigm (Purves et al. 1995) were essentially identical to those of a between-subject experiment that also used permanent lesions (Reilly et al. 1993). Finally, there is no evidence of inter-stimulus "competition" in our data set, nor of MUS effects transferring from one taste to the other. Thus, the most important differences between our study and earlier studies probably have to do with our relatively small aversion effects and our use of muscimol.

If conditioned taste aversions are learned supra-optimally when the hippocampus is incapacitated, the implication is that a normally functioning hippocampus may interfere with the learning of a CTA. While this corollary is difficult to test, it is consistent with the findings of Packard and colleagues, who have suggested that normal hippocampal function interferes with striataldependent learning processes (Poldrack and Packard 2003). These researchers trained normal and lesioned rats to perform radial arm maze tasks in which spatial cues either indicated or were irrelevant to the arm that had been baited with food (this latter task required that the rats learn the "habit" of turning in a particular direction at the maze center-learning that is striataldependent), and reported that hippocampal lesions impaired spatial learning while enhancing habit learning (Schroeder et al. 2002).

Could a similar process be occurring here? The hippocampus is known to be involved in spatial foraging (Baird Day et al. 1999; Pravosudov and Clayton 2002), but it seems doubtful that spatial learning processes actually compete and interfere with chemosensory learning processes in our one-bottle instantiation of the CTA paradigm, at least not in the same way that spatial and habit learning processes interfere with each other when a rat must choose a lane in a radial arm maze (Poldrack and Packard 2003). It does not appear that rats use spatial information to ensure avoidance of a toxin even when that spatial information is the only viable cue (Mediavilla et al. 2001). Still, perhaps the hippocampus' "attempt" to map spatial locations onto food sources interferes with the explicitly non-spatial task, in this case amygdalar learning of stimulus palatability. It is known that lesions of the amygdala (which impair CTA; see Rolls et al. 1989; Lamprecht et al. 1997; Schafe et al. 1998; Morris et al. 1999; Yasoshima et al. 2000; Reilly and Bornovalova 2005) affect the strategies that rats use in maze learning tasks (Packard and Wingard 2004). This fact is consistent with the possibility that the CTA machinery and hippocampus may interact in some way.

At another level, the observed learning enhancement may be a function of the complexities of the hippocampus' relationship with the distributed dopamine system, which is involved in learning about stimulus "value" (Berridge and Robinson 1998). In many circumstances, the hippocampal projections to the dopamine system work in opposition to striatal, prefrontal, and

\section{Learning \& Memory}


A

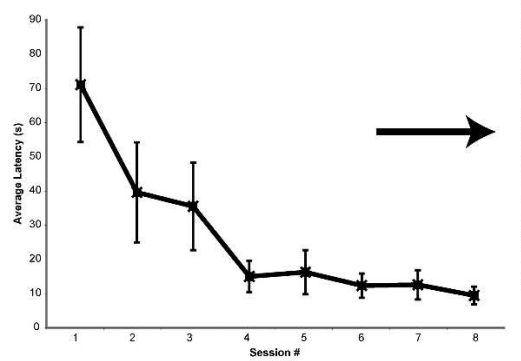

$\mathrm{B}$

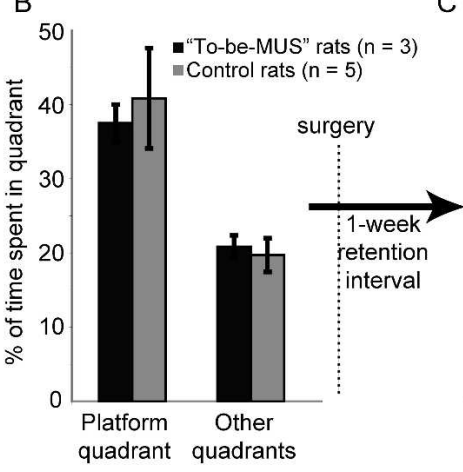

C

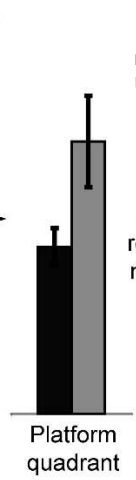

$\mathrm{D}$

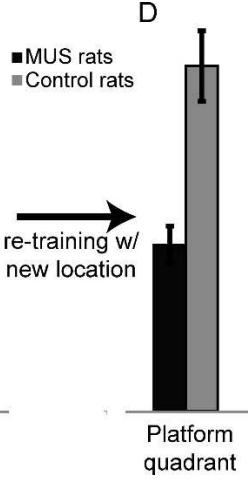

Figure 6. Inactivation of dorsal hippocampus impairs spatial learning. (A) In a replication of Moser and Moser (1998), rats were first trained in the hidden-platform version of the Morris Water Maze task. ( $x$-axis) Session, ( $y$-axis) latency to find the platform. Rats learned the task in four sessions, as evidenced by the gradual asymptote in speed of finding the platform. (B) The standard transfer task, in which the platform is removed before the rats enter the water, showed that rats had learned to spend the bulk of their time searching for the platform in the appropriate quadrant (where it had been before removal), relative to the average time spent in other quadrants. For this and all subsequent panels, the $y$-axis is percentage of total swimming time. All rats were identical at this point, but we have divided them into two groups to demonstrate the lack of initial differences between the rats that would later receive MUS inactivation of dHIPP (dark bars) and those that would not (light bars). Following performance on the transfer task, rats received surgery; 1 wk of recovery separated panel $B$ from panel C. (C) Retention, tested with the transfer task, was relatively good for control rats (light bar), but rats with inactivated hippocampi (dark bar) spent relatively little time in the appropriate quadrant. $(D)$ Subsequent retraining with the platform in a new spatial location, again tested with a subsequent transfer task, went quickly for control rats (light bar). Following hippocampal inactivation (dark bar), however, rats did not learn to spend time in the appropriate quadrant.

amygdalar dopamine projections (Simon et al. 1988; Lipska et al. 1992; Joseph et al. 2000). Furthermore, hippocampectomy affects this system in ways that evolve, recover, and even change sign over time (Lanier et al. 1975; Hannigan Jr. et al. 1984; Lipska et al. 1992). Perhaps hippocampal inactivation causes changes in the accumbens dopamine circuit that are countered by opposing changes in taste-related branches of the dopamine circuitry (a related argument has been made with regard to latent inhibition; see Ellenbroek et al. 1997); these latter changes would be expected to reverse the typical damaging effect of the hippocampal lesion. The fact that hippocampal lesions eliminate the contextspecificity of latent inhibition in fear conditioning and conditioned emotional response paradigms (Honey and Good 1993; Holt and Maren 1999) corroborates this suggestion, demonstrating that learning phenomena may appear in novel situations following hippocampectomy.

Our study suggests that hippocampal involvement in learning may be highly context-dependent, changing with the techniques and paradigms used, and the above discussion has necessarily kept that technique-dependence in mind. Any explanation of hippocampal involvement in CTA on the basis of our findings would necessarily be premature and difficult to generalize. Still, we can think of several (admittedly speculative) ways in which the two main findings-hippocampal inactivation enhancing latent inhibition, and hippocampal inactivation enhancing learning - can be explained in a unified fashion. If, for instance, the impact of the inactivation was to increase stimulus salience (say, by reducing the attention paid to context), then that increased stimulus salience would enhance whatever learning the rat was engaged in at that time. Alternatively, if one considers time as a facet of context that is processed by the hippocampus (as is made explicit by hippocampal theories of sequence learning; see Fortin et al. 2002; Eichenbaum 2004; Kesner and Rogers 2004), then it is possible to think of hippocampal inactivation as preventing the rat from recognizing the change between training and testing context that accrues with the passage of time. This increase in similarity between the two contexts would enhance whatever learning (latent inhibition or CTA) took place during inactivation. Finally, it is possible that our findings have to do with stress, which can affect learning (Abidin et al. 2004) and is itself affected by hippocampal manipulations (Feldman and Weiden- feld 2001); it is not clear, however, that dorsal hippocampal (as opposed to ventral; see Kjelstrup et al. 2002; Laplante et al. 2004) lesions actually affect stress appropriately for improved performance, nor is this explanation clearly consistent with the large corpus of studies on the hippocampus and learning.

Whatever explanation proves useful and valid, the enhancement of CTA via hippocampal inactivation makes it clear that hippocampal and amygdalar memory circuits, while performing different functions, do not function in isolation from each other. The recursive connectivity among limbic and associated structures (Pare et al. 2002) implies that activity in the hippocampal network has an impact on processing in other memory systems, even when the hippocampus is unnecessary for learning. A complete understanding of the neural substrates of learning and memory will require an understanding of the interactions between these systems.

\section{Materials and Methods}

\section{Subjects}

All methods conform to Brandeis University's animal use and care guidelines. Studies were performed using female Long-Evans rats (Charles River Laboratories) with body weights of 250-320 g at the time of surgery; while female rats condition somewhat more consistently than male rats (Chambers et al. 1981), this fact in no way interferes with our results. Subjects were housed individually in an animal facility with a $12 \mathrm{~h}$ light/12 h dark cycle (lights out at 7 p.m.) with ad libitum access to standard chow. Experiments were run in the afternoon in squads of four.

\section{Surgery}

Rats were anesthetized via intraperitoneal (ip) injection (ketamine, $100 \mathrm{mg} / \mathrm{kg}$; xylazine, $5 \mathrm{mg} / \mathrm{kg}$; acepromozine, $1 \mathrm{mg} / \mathrm{kg}$ ) and given update doses of ketamine ip as needed to maintain deep anesthesia, determined by breathing rate and response to foot pinch. Subjects were placed in a stereotaxic frame, the scalp was incised and retracted, and the skull was leveled. Small holes were drilled into the skull for bilateral placement of guide cannulae (23-gauge, $15 \mathrm{~mm}$ in length) in the dorsal hippocampus (dHIPP, coordinates relative to bregma: AP $-3.8 \mathrm{~mm}, \mathrm{ML} \pm 2.5$ $\mathrm{mm}, \mathrm{DV}-1.8 \mathrm{~mm}$ from dura; $\mathrm{n}=28$ ); muscimol infused at these single sites is sufficient to impair performance of a spatial learning task (Moser and Moser 1998). A subset of animals also re- 
ceived guide cannulae placed in the taste-responsive region (Kosar et al. 1986; Katz et al. 2001) of insular cortex (GC, AP +1.4 $\mathrm{mm}, \mathrm{ML} \pm 5 \mathrm{~mm}, \mathrm{DV}-2.5 \mathrm{~mm}$ from dura; $\mathrm{n}=9$ ). Cannulae were anchored to the skull with four 0-80 screws and dental acrylic. Stainless steel stylets (30-gauge, $15 \mathrm{~mm}$ in length) were inserted into the guide cannulae to ensure patency.

\section{Training protocol}

Following 1 wk of recovery from surgery, rats began a training regimen wherein each was familiarized to one tastant (taste-fam) and conditioned to both that tastant and a novel tastant (tastenov). First, rats were acclimated to taking fluids in a testing chamber with a single lick spout in one end. After adaptation sessions, they were familiarized with taste-fam; such repeated taste pre-exposures lead to latent inhibition (LI)-later difficulty associating the taste with sickness. Next they were trained, using two pairings of each taste with LiCl-induced gastric distress. The learning was evaluated in taste-alone tests: One taste was presented at the single lick spout, the rats' desire to drink was recorded, and no LiCl was delivered.

In more detail, the protocol was as follows (as schematized in Table 1):

\section{Days 1-4 (acclimation)}

Rats were trained to obtain a daily fluid ration in two drinking periods per day. Afternoon 30-min sessions took place shortly after noon, in experimental chambers located in a soundattenuating enclosure. For these sessions, rats were given $30 \mathrm{~min}$ to consume $10 \mathrm{~mL}$ of water from a single lick spout, after which they were returned to their home cages in the animal facility. At midnight, animals received $15 \mathrm{~min}$ of ad lib access to water in their home cages (this midnight session continued through all subsequent days). The midnight feeding, delivered during the rats' active period, ensured that rats never went more than $\sim 12 \mathrm{~h}$ without water, but also ensured that they were very thirsty for the actual sessions.

\section{Days 5-8 (familiarization)}

Rats received either VEH or MUS into bilateral dHIPP $30 \mathrm{~min}$ prior to being moved to the experimental chamber for their afternoon drinking sessions; for these sessions, rats were given 30 min to consume $10 \mathrm{~mL}$ of taste-fam.

\section{Days 9-12 (conditioning)}

Rats received bilateral infusions of either VEH or MUS into dHIPP $30 \mathrm{~min}$ prior to being moved to the experimental chambers for their afternoon drinking sessions. For these sessions, they were given $30 \mathrm{~min}$ access to $10 \mathrm{~mL}$ of either taste-fam or taste-nov at the single lick spout. Immediately following this session, animals received $10 \mathrm{~mL} / \mathrm{kg}$ of $0.15 \mathrm{M} \mathrm{LiCl}$ via ip injection in their home cages; this injection was sufficient to induce visual evidence of gastric distress. In total there were two conditioning sessions for each tastant, delivered in alternation (i.e., taste-nov on Day 9, taste-fam on Day 10, taste-nov on Day 11, and taste-fam on Day 12).

\section{Days 13-14 (testing)}

Rats were returned to the experimental chambers for their afternoon drinking sessions, where they were given $30 \mathrm{~min}$ ad lib

Table 1. Day-by-day description of training

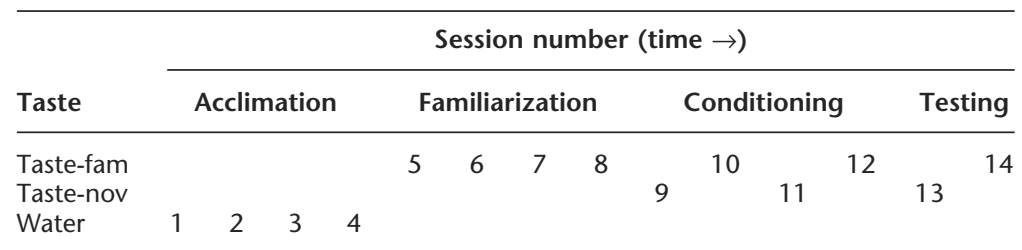

The order of conditioning and testing trials was counterbalanced, such that for half of the rats session 9 was the first taste-fam conditioning session, etc. access (i.e., in these sessions the rats could conceivably drink $>10$ $\mathrm{mL}$ ) to either taste-fam or taste-nov at the single lick spout. One taste was tested in each session.

\section{Intra-cranial infusions}

The infusion procedure was adapted from Holt and Maren (1999). Stylets were removed and 30-gauge infusion cannulae (15 $\mathrm{mm}$ long for dHIPP infusion; $17 \mathrm{~mm}$ long for GC infusion, for final DV coordinate $-4.5 \mathrm{~mm}$ ) connected via PE-10 tubing to 10- $\mu \mathrm{L}$ Hamilton syringes mounted in a dual syringe pump (Harvard Apparatus) were inserted into the guide cannulae. $0.5 \mu \mathrm{L}$ of either $1 \mu \mathrm{g} / \mathrm{\mu L}$ muscimol (MUS: 5-(Aminomethyl)-3-isoxazolol; Sigma) in $0.9 \%$ saline or the $0.9 \%$ saline vehicle (VEH) alone was infused bilaterally over $60 \mathrm{sec}$; these infusions hyperpolarize, and thus effectively cease the firing of, virtually any neurons in the vicinity with GABA synapses (Krupa et al. 1999). During infusions, rats sat in the experimenter's lap and were discouraged from grooming or excessive motion. Infusion cannulae were left in place for $2 \mathrm{~min}$ post-infusion to ensure that the liquid had diffused from the injection site, and the stylets were replaced.

Rats were randomly assigned to groups that differed with regard to hippocampal infusant, muscimol (MUS) or vehicle (VEH). Groups, as summarized in Table 2, included the following: Some received MUS infusions during the familiarization phase only, while others received MUS infusions during conditioning sessions only (Fig. 2); in addition, some received MUS during both familiarization and conditioning sessions (Fig. 4). Two infusion control groups were run: One received VEH infusions before familiarization and conditioning sessions, and one received no infusions at all.

Finally, sham-conditioned (taste paired with ip saline injections) controls allowed us to examine drinking in rats that had been subjected to otherwise identical training.

\section{Stimuli}

The tastants used were $0.1 \mathrm{M} \mathrm{NaCl}$ and $0.1 \mathrm{M}$ sucrose, chosen to facilitate comparison with electrophysiological studies (e.g., Katz et al. 2001). Pilot studies revealed that rats preferred these concentrations of sucrose and $\mathrm{NaCl}$ over water (although not to precisely the same degree) in a two-bottle test. Each tastant served as taste-fam for half of the subjects in each group; while taste was counterbalanced, this variable had no impact on the results, and the data from rats for which $\mathrm{NaCl}$ served as taste-fam were combined with the data from rats in the same group for which sucrose served as taste-fam.

\section{CTA training with cortical inactivation}

One group of rats, including a subset of rats from the dHIPP study, were subsequently run in a simpler CTA paradigm in the same apparatus, to test that our infusion and training procedures produced expected effects. The tastant used was $0.02 \mathrm{M}$ citric acid (which was not preferred over water, but which was not highly unpalatable). The procedures used were a subset of those used for the two-taste experiment; no familiarization sessions were run, and rats received only one conditioning and one testing session. Subjects were randomly assigned to one of two groups, differing with regard to cortical infusant, VEH or MUS. Consumption of these rats was further compared with drinking in a "pseudoconditioned" (unpaired presentation of taste and $\mathrm{LiCl}$ ) group.

\section{Quantification and analysis of CTA learning}

Amount of fluid consumed in acclimation, familiarization, conditioning, and testing sessions was quantified to the nearest $0.5 \mathrm{~mL}$. CTA learning could be recognized as a reduction (from baseline) in the amount of tastant consumed, and latent inhibition could be recognized in the difference between the amount of taste-fam and taste-nov consumed. The significance of any differences in consumption was evaluated using $t$-tests and mixed effects anovae during the single testing sessions, as described in the Results section, using the statistics package S-plus (In- 
Hippocampus and taste learning

Table 2. Infusion schedule for the five dHIPP and CTA groups

dHIPP infusant received ( $1 / 2 \mathrm{hr}$ before tasting session)

\begin{tabular}{llll}
\cline { 3 - 4 } Group & Surgery & Familiarization (4 d) & Conditioning (4 d) \\
\hline Group 1 (dHIPP \& familiarization) & dHIPP cannulae & Muscimol (MUS) & Vehicle (VEH) \\
Group 2 (dHIPP \& taste learning) & dHIPP cannulae & VEH & MUS \\
Group 3 ("virtual lesion") & dHIPP cannulae & MUS & MUS \\
Group 4 (vehicle control) & dHIPP cannulae & VEH & VEH \\
Group 5 (unoperated control) & none & & \\
\hline
\end{tabular}

Groups 1 and 2 provided data for Figure 2. Group 3 provided data for Figure 4 . Groups 4 and 5 provided data for both figures. (dHIPP) Dorsal hippocampus, (CTA) conditioned taste aversion, (MUS) muscimol, (VEH) vehicle.

sightful Corp). Results for conditioning sessions were qualitatively identical.

\section{Morris Water Maze training with dHIPP muscimol infusion}

Our water maze test was similar to that described by the Moser group (Moser and Moser 1998). Rats were trained to swim through water made opaque by the addition of white, nontoxic paint to a submerged platform in a $150-\mathrm{cm}$ diameter pool surrounded by walls with large, high-contrast black and white pictures. The submerged platform was consistently placed from trial to trial for each rat (but differently placed for different rats), such that the location of the platform was learnable in relation to the distal spatial cues.

In each trial the rat was dropped into the pool, facing the wall. The starting point was selected pseudo-randomly, such that each rat eventually started at each of eight points around the perimeter in eight trials. Once in the water, the rat was given 120 sec to find the platform on its own; failing that, it was guided to the platform by hand, and allowed to rest there for 5 sec until the next trial. Latency to find the platform was noted with a timer and verified via digital recordings. Each rat received eight trials per day (two sessions of four trials, with 30 min between session) across 8 days, for a total of 32 trials.

On the day following the completion of training, rats received a single probe trial without a platform. The platform was removed from the pool, and the rat was released in a spot opposite to the previous location of the platform. The rat's search pattern across the subsequent $20 \mathrm{sec}$ was digitally recorded; the percentage of time spent in each quadrant was later coded by an undergraduate blind to condition, and the percentage of time spent in the quadrant with the platform was used as an index of learning. Following this trial, the animal was removed from the pool, the platform was replaced, and the animal was placed once more into the pool and allowed to locate the platform.

Next, the rats were surgically prepared as for the CTA experiment, and after recovery from surgery the probe trial was repeated (this trial was called the "retention test"), $15 \mathrm{~min}$ following MUS (or control) infusions into dHIPP. Such infusions have been previously shown to disrupt probe trial performance (Moser and Moser 1998). Immediately following this second probe trial, the distal cues were rearranged (disrupting any of the originally learned spatial relationships), the platform was placed in the pool in a new location, and the infused rats were retrained across four more blocks of two trials. Blocks were separated by 5 min. Finally, a third probe trial (the "relearning test") assessed whether inactivation of dHIPP interfered with new spatial learning.

\section{Histology}

Cannula placement was confirmed histologically after the experiment. Rats were deeply anaesthetized and perfused transcardially with $10 \%$ saline followed by $10 \%$ formol-saline. Brains were removed and refrigerated in 30\% sucrose/10\% formalin solution for several days. Coronal sections $(40 \mu \mathrm{m})$ were cut on a cryostat and mounted to slides. Sections were stained with cresyl violet to visualize cannula tracks.

\section{Acknowledgments}

This work was funded by NIDCD grant 5R03-DC005312 and by Brandeis start-up funds. We thank Jill Crocker and Jenn Yunyan Wang for feeding and toweling off the rats, and Jenn Yunyan Wang for additional histology. We also thank Asif Ghazanfar, Leslie Griffith, and Gina Turrigiano for helpful comments.

\section{References}

Abidin, I., Yargicoglu, P., Agar, A., Gumuslu, S., Aydin, S., Ozturk, O., and Sahin, E. 2004. The effect of chronic restraint stress on spatial learning and memory: Relation to oxidant stress. Int. J. Neurosci. 114: $683-699$.

Baird Day, L., Crews, D., and Wilczynski, W. 1999. Relative medial and dorsal cortex volume in relation to foraging ecology in congeneric lizards. Brain Behav. Evol. 54: 314-322.

Bannerman, D.M., Yee, B.K., Good, M.A., Heupel, M.J., Iversen, S.D., and Rawlins, J.N. 1999. Double dissociation of function within the hippocampus: A comparison of dorsal, ventral, and complete hippocampal cytotoxic lesions. Behav. Neurosci. 113: 1170-1188.

Bast, T., Zhang, W.N., and Feldon, J. 2003. Dorsal hippocampus and classical fear conditioning to tone and context in rats: Effects of local NMDA-receptor blockade and stimulation. Hippocampus 13: $657-675$.

Bellingham, W.P., Gillette-Bellingham, K., and Kehoe, E.J. 1985. Summation and configuration in patterning schedules with the rat and rabbit. Anim. Learn. Behav. 13: 152-164.

Berridge, K.C. and Robinson, T.E. 1998. What is the role of dopamine in reward: Hedonic impact, reward learning, or incentive salience? Brain Res. Brain Res. Rev. 28: 309-369.

Bielavska, E. and Roldan, G. 1996. Ipsilateral connections between the gustatory cortex, amygdala and parabrachial nucleus are necessary for acquisition and retrieval of conditioned taste aversion in rats. Behav. Brain Res. 81: 25-31.

Bures, J., Bermúdez-Rattoni, F., and Yamamoto, T. 1998. Conditioned taste aversion: Memory of a special kind. Oxford University Press, Oxford, UK.

Chambers, K.C., Sengstake, C.B., Yoder, R.L., and Thornton, J.E. 1981. Sexually dimorphic acquisition of a conditioned taste aversion in rats: Effects of gonadectomy, testosterone replacement and water deprivation. Physiol. Behav. 27: 83-88.

Coutureau, E., Galani, R., Gosselin, O., Majchrzak, M., and Di Scala, G. 1999. Entorhinal but not hippocampal or subicular lesions disrupt latent inhibition in rats. Neurobiol. Learn. Mem. 72: 143-157.

Eichenbaum, H. 2004. Hippocampus: Cognitive processes and neural representations that underlie declarative memory. Neuron 44: $109-120$.

Ellenbroek, B.A., Knobbout, D.A., and Cools, A.R. 1997. The role of mesolimbic and nigrostriatal dopamine in latent inhibition as measured with the conditioned taste aversion paradigm. Psychopharmacology 129: 112-120.

Feldman, S. and Weidenfeld, J. 2001. Electrical stimulation of the dorsal hippocampus caused a long lasting inhibition of ACTH and adrenocortical responses to photic stimuli in freely moving rats. Brain Res. 911: 22-26.

Fortin, N.J., Agster, K.L., and Eichenbaum, H.B. 2002. Critical role of the hippocampus in memory for sequences of events. Nat. Neurosci. 5: $458-462$.

Gallo, M. and Candido, A. 1995. Dorsal hippocampal lesions impair 
blocking but not latent inhibition of taste aversion learning in rats. Behav. Neurosci. 109: 413-425.

Gallo, M., Roldan, G., and Bures, J. 1992. Differential involvement of gustatory insular cortex and amygdala in the acquisition and retrieval of conditioned taste aversion in rats. Behav. Brain Res. 52: 91-97.

Grecksch, G., Bernstein, H.G., Becker, A., Hollt, V., and Bogerts, B. 1999. Disruption of latent inhibition in rats with postnatal hippocampal lesions. Neuropsychopharmacology 20: 525-532.

Gutierrez, R., Rodriguez-Ortiz, C.J., De La Cruz, V., Nunez-Jaramillo, L., and Bermudez-Rattoni, F. 2003. Cholinergic dependence of taste memory formation: Evidence of two distinct processes. Neurobiol. Learn. Mem. 80: 323-331.

Hall, G. and Minor, H. 1984. A search for context-stimulus associations in latent inhibition. Q. J. Exp. Psychol. 36B: 145-169.

Hannigan Jr., J.H., Springer, J.E., and Isaacson, R.L. 1984. Differentiation of basal ganglia dopaminergic involvement in behavior after hippocampectomy. Brain Res. 291: 83-91.

Holt, W. and Maren, S. 1999. Muscimol inactivation of the dorsal hippocampus impairs contextual retrieval of fear memory. $J$. Neurosci. 19: 9054-9062.

Honey, R.C. and Good, M. 1993. Selective hippocampal lesions abolish the contextual specificity of latent inhibition and conditioning. Behav. Neurosci. 107: 23-33.

Joseph, M.H., Peters, S.L., Moran, P.M., Grigoryan, G.A., Young, A.M., and Gray, J.A. 2000. Modulation of latent inhibition in the rat by altered dopamine transmission in the nucleus accumbens at the time of conditioning. Neuroscience 101: 921-930.

Katz, D.B., Simon, S.A., and Nicolelis, M.A. 2001. Dynamic and multimodal responses of gustatory cortical neurons in awake rats. $J$. Neurosci. 21: 4478-4489.

Kaye, H. and Pearce, J.M. 1987. Hippocampal lesions attenuate latent inhibition and the decline of the orienting response in rats. Q. J. Exp. Psychol. B 39: 107-125.

Kehoe, E.J., Horne, P.S., and Horne, A.J. 1993. Discrimination learning using different CS-US intervals in classical conditioning of the rabbit's nictitating membrane response. Psychobiology 21: 277-285.

Kesner, R.P. and Rogers, J. 2004. An analysis of independence and interactions of brain substrates that subserve multiple attributes, memory systems, and underlying processes. Neurobiol. Learn. Mem. 82: 199-215.

Kjelstrup, K.G., Tuvnes, F.A., Steffenach, H.A., Murison, R., Moser, E.I., and Moser, M.B. 2002. Reduced fear expression after lesions of the ventral hippocampus. Proc. Natl. Acad. Sci. 99: 10825-10830.

Kosar, E., Grill, H.J., and Norgren, R. 1986. Gustatory cortex in the rat. I. Physiological properties and cytoarchitecture. Brain Res. 379: 329-341.

Krupa, D.J., Ghazanfar, A.A., and Nicolelis, M.A.L. 1999. Immediate thalamic sensory plasticity depends on corticothalamic feedback. Proc. Natl. Acad. Sci. 96: 8200-8205.

Lamprecht, R., Hazvi, S., and Dudai, Y. 1997. cAMP response element-binding protein in the amygdala is required for long- but not short-term conditioned taste aversion memory. J. Neurosci. 17: 8443-8450.

Lanier, L.P., Van Hartesveldt, C., Weis, B.J., and Isaacson, R.L. 1975. Effects of differential hippocampal damage upon rhythmic and stress-induced corticosterone secretion in the rat. Neuroendocrinology 18: $154-160$.

Laplante, F., Stevenson, C.W., Gratton, A., Srivastava, L.K., and Quirion, R. 2004. Effects of neonatal ventral hippocampal lesion in rats on stress-induced acetylcholine release in the prefrontal cortex. $J$. Neurochem. 91: 1473-1482.

Lasiter, P.S., Deems, D.A., and Garcia, J. 1985. Involvement of the anterior insular gustatory neocortex in taste-potentiated odor aversion learning. Physiol. Behav. 34: 71-77.

Lipska, B.K., Jaskiw, G.E., Chrapusta, S., Karoum, F., and Weinberger, D.R. 1992. Ibotenic acid lesion of the ventral hippocampus differentially affects dopamine and its metabolites in the nucleus accumbens and prefrontal cortex in the rat. Brain Res. 585: 1-6.

Mediavilla, C., Molina, F., and Puerto, A. 2001. Effects of a flavor-placement reversal test after different modalities of taste aversion learning. Neurobiol. Learn. Mem. 76: 209-224.

Morris, R., Frey, S., Kasambira, T., and Petrides, M. 1999. Ibotenic acid lesions of the basolateral, but not the central, amygdala interfere with conditioned taste aversion: Evidence from a combined behavioral and anatomical tract-tracing investigation. Behav. Neurosci. 113: 291-302.

Moser, M.B. and Moser, E.I. 1998. Distributed encoding and retrieval of spatial memory in the hippocampus. J. Neurosci. 18: 7535-7542.

Naor, C. and Dudai, Y. 1996. Transient impairment of cholinergic function in the rat insular cortex disrupts the encoding of taste in conditioned taste aversion. Behav. Brain Res. 79: 61-67.

Packard, M.G. and Wingard, J.C. 2004. Amygdala and "emotional" modulation of the relative use of multiple memory systems. Neurobiol. Learn. Mem. 82: 243-252.

Pare, D., Collins, D.R., and Pelletier, J.G. 2002. Amygdala oscillations and the consolidation of emotional memories. Trends Cogn. Sci. 6: $306-314$.

Paxinos, G. and Watson, C. 1997. The rat brain in stereotaxic coordinates. Academic Press, San Diego, CA.

Poldrack, R.A. and Packard, M.G. 2003. Competition among multiple memory systems: Converging evidence from animal and human brain studies. Neuropsychologia 41: 245-251.

Pravosudov, V.V. and Clayton, N.S. 2002. A test of the adaptive specialization hypothesis: Population differences in caching, memory and the hippocampus in black-capped chickadees (Poecile atricapilla). Behav. Neurosci. 16: 515-522.

Purves, D., Bonardi, C., and Hall, G. 1995. Enhancement of latent inhibition in rats with electrolytic lesions of the hippocampus. Behav. Neurosci. 109: 366-370.

Reilly, S. and Bornovalova, M.A. 2005. Conditioned taste aversion and amygdala lesions in the rat: A critical review. Neurosci. Biobehav. Rev. 29: 1067-1088.

Reilly, S., Harley, C., and Revusky, S. 1993. Ibotenate lesions of the hippocampus enhance latent inhibition in conditioned taste aversion and increase resistance to extinction in conditioned taste preference. Behav. Neurosci. 107: 996-1004.

Rolls, E.T., Sienkiewicz, Z.J., and Yaxley, S. 1989. Hunger modulates the responses to gustatory stimuli of single neurons in the caudolateral orbitofrontal cortex of the macaque monkey. Eur. J. Neurosci. 1: $53-60$.

Santini, E., Muller, R.U., and Quirk, G.J. 2001. Consolidation of extinction learning involves transfer from NMDA-independent to NMDA-dependent memory. J. Neurosci. 21: 9009-9017.

Schafe, G.E. and Bernstein, I.L. 1998. Forebrain contribution to the induction of a brainstem correlate of conditioned taste aversion. II. Insular (gustatory) cortex. Brain Res. 800: 40-47.

Schafe, G.E., Thiele, T.E., and Bernstein, I.L. 1998. Conditioning method dramatically alters the role of amygdala in taste aversion learning. Learn. Mem. 5: 481-492.

Schmajuk, N.A., Lam, Y.-W., and Christiansen, B.A. 1994. Latent inhibition of the rat eyeblink response: Effect of hippocampal aspiration lesions. Physiol. Behav. 55: 597-601.

Schroeder, J.P., Wingard, J.C., and Packard, M.G. 2002. Post-training reversible inactivation of hippocampus reveals interference between memory systems. Hippocampus 12: 280-284.

Shohamy, D., Allen, M.T., and Gluck, M.A. 2000. Dissociating entorhinal and hippocampal involvement in latent inhibition. Behav. Neurosci. 114: 867-874.

Simon, H., Taghzouti, K., Gozlan, H., Studler, J.M., Louilot, A., Herve, D., Glowinski, J., Tassin, J.P., and Le Moal, M. 1988. Lesion of dopaminergic terminals in the amygdala produces enhanced locomotor response to D-amphetamine and opposite changes in dopaminergic activity in prefrontal cortex and nucleus accumbens. Brain Res. 447: 335-340.

Spector, A.C. 1995. Gustatory function in the parabrachial nuclei: Implications from lesion studies in rats. Rev. Neurosci. 6: 143-175.

Yamamoto, T., Fujimoto, Y., Shimura, T., and Sakai, N. 1995. Conditioned taste aversion in rats with excitotoxic brain lesions. Neurosci. Res. 22: 31-49.

Yasoshima, Y. and Yamamoto, T. 1997. Rat gustatory memory requires protein kinase $\mathrm{C}$ activity in the amygdala and cortical gustatory area. Neuroreport 8: 1363-1367.

Yasoshima, Y., Morimoto, T., and Yamamoto, T. 2000. Different disruptive effects on the acquisition and expression of conditioned taste aversion by blockades of amygdalar ionotropic and metabotropic glutamatergic receptor subtypes in rats. Brain Res. 869: $15-24$.

Yee, B.K., Feldon, J., and Rawlins, J.N.P. 1995. Latent inhibition in rats is abolished by NMDA-induced neuronal loss in the retrohippocampal region, but this lesion effect can be prevented by systemic haloperidol treatment. Behav. Neurosci. 109: 227-240.

Received July 17, 2005; accepted in revised form August 16, 2005. 


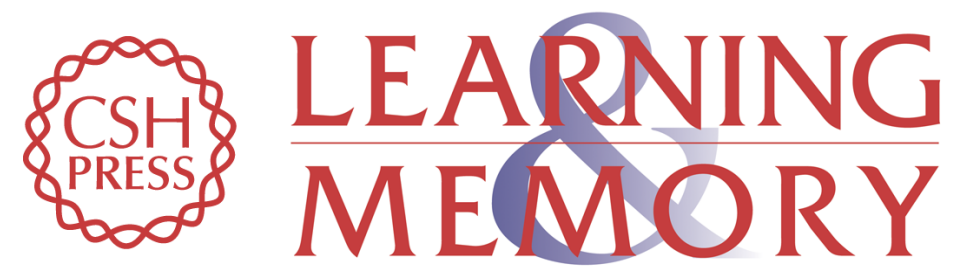

\section{Hippocampal inactivation enhances taste learning}

Martha E. Stone, Brandon S. Grimes and Donald B. Katz

Learn. Mem. 2005, 12:

Access the most recent version at doi:10.1101//m.32305

References This article cites 57 articles, 8 of which can be accessed free at: http://learnmem.cshlp.org/content/12/6/579.full.html\#ref-list-1

License

Email Alerting Receive free email alerts when new articles cite this article - sign up in the box at the Service top right corner of the article or click here. 